\title{
'|||||||||||||||||||||||||||||||||||||||||||||||||||||||||||||||||.
}

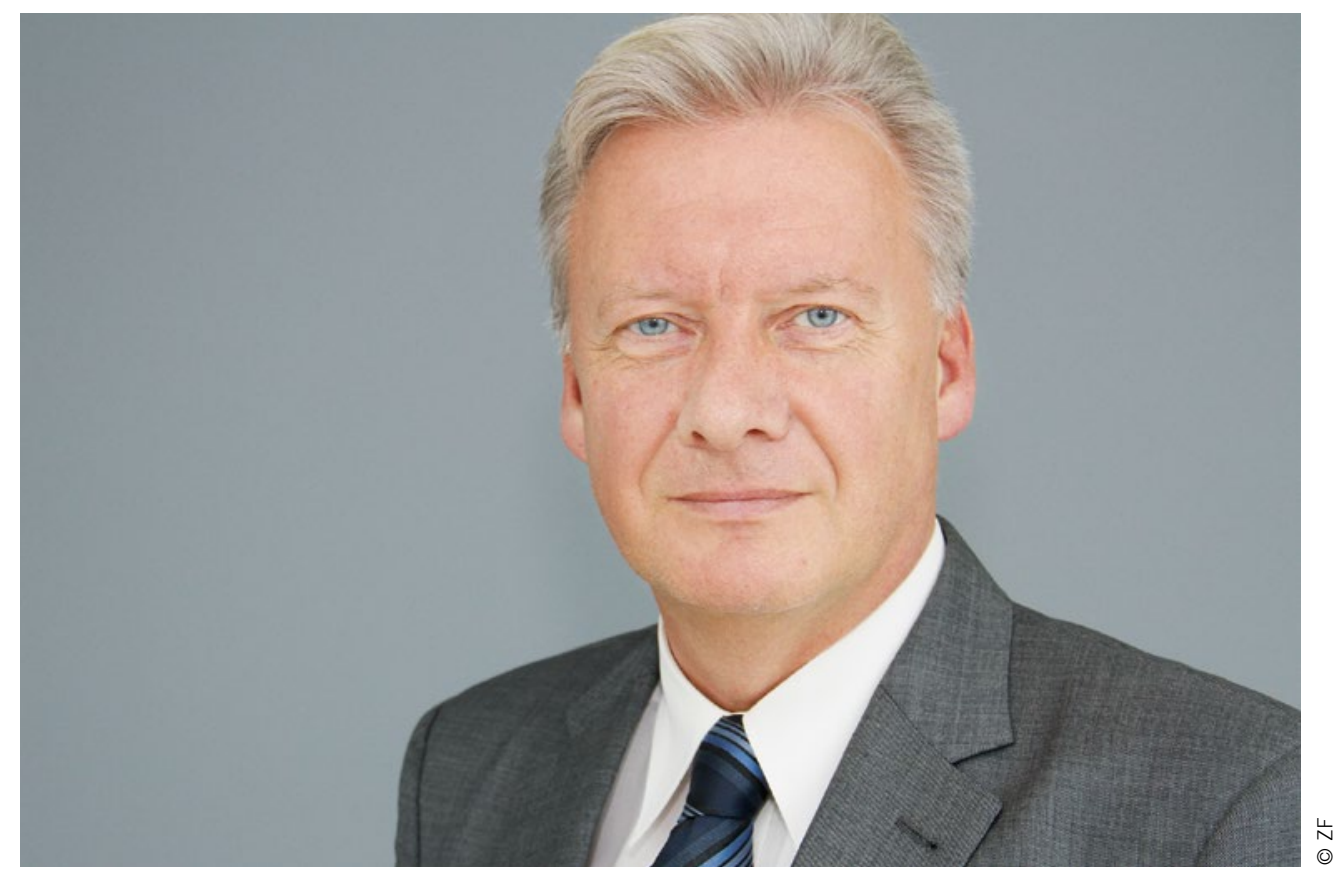

Udo Kneitz

Senior Vice President Industrial Technology und Off-Highway Systems der ZF Friedrichshafen AG in Passau

\section{Intelligente mechanische Systeme}

Digitalisierung und Vernetzung sind mittlerweile fester Bestandteil unseres täglichen Lebens. Via Smartphone stellen wir Inhalte über Social Media und Clouds zur Verfügung und können diese ebenso jederzeit abrufen. Unsere Autos bieten immer intelligentere Funktionen, um uns gezielt zu entlasten und das Fahren dadurch sicherer zu machen. Elektrifizierung und autonomes Fahren im Pkw und Nkw sind greifbar nahe. Aber auch in den Bereichen Material-Handling, Bau- oder Landmaschine lassen sich diese Trends bestätigen. Hier arbeiten wir gleichermaßen an marktgerechten Lösungen.

Die zunehmende Urbanisierung ist ein wesentlicher Treiber hin zu emissionslosen und effizienteren Antriebssystemen im Bereich Offhighway. Maschinen werden zukünftig weniger genutzt, um neue Infrastrukturen im Bereich der Ballungszentren aufzubauen, sondern vielmehr, um bereits bestehende Bauten zu erhalten und instand zu setzen. Um in Zukunft in Städten lärm- und emissionslos arbeiten zu können, werden sich, letztlich auch auf Druck von Regierungen und Behörden, die entsprechenden Technologien wandeln müssen. Nichtsdestoweniger werden konventionelle Antriebslösungen noch lange in unseren Märkten Bestand haben. Elektrische und automatisierte Fahrzeuge werden sich parallel dazu schrittweise etablieren - diese Entwicklung sehen wir als logische Evolution - nicht als kurzfristig einsetzende Revolution.

Unsere Kunden denken und handeln immer globaler. Sie erwarten, dass ihre Zulieferer dieser Strategie zur weltweiten Präsenz folgen und die Produkte den Anforderungen der Märkte entspre- chend flexibel und zeitnah anpassen. Um dem harten Wettbewerb bei gleichzeitig gesteigerten Kundenwünschen gerecht zu werden, müssen wir modulare Systeme entwickeln, mit denen wir die gewünschten Technologie-Level gleichermaßen bedienen können.

Im ersten Schritt können Effizienz und Produktivität aller Antriebskomponenten weiter optimiert werden. Hier wird neben der CVT-Technologie auch die Automatisierung eine Schlüsselrolle spielen, um den zukünftigen Marktanforderungen gerecht zu werden. Im zweiten Schritt sehen wir Elektrifizierung und autonomes Fahren als die Megatrends in der Personenbeförderung, welche auch im Bereich Offhighway in einer Vielzahl von Applikationen zur Anwendung kommen werden. Gemeinsam mit unseren Kunden sind wir in der Lage, diese Zukunft zu gestalten und leistungsfähige, intelligente mechanische, aber auch elektrische und autonome Systeme zu entwickeln. Es bedarf also neuer Produkte und natürlich auch innovativer Produktionsweisen, um diesen globalen Technologietrends in geeigneter Weise folgen zu können.

Es ist unser erklärtes Ziel, mit zukunftsweisenden Technologien und Funktionen die Fahrzeuge unserer Kunden effizienter, sicherer und wartungsfreundlicher zu machen. Zudem nutzen wir im Bereich der ZF-Industrietechnik gezielt die Potenziale, die sich aus dem Einsatz künstlicher Intelligenz und korrelierender Big-Data- und Deep-Learning-Anwendungen ableiten lassen. So können wir unsere Flexibilität, unser Innovationspotenzial und unsere Innovationsgeschwindigkeit im Sinne der Kunden kontinuierlich steigern. 